\title{
Coulomb corrections to inclusive cross sections at the future Electron-Ion Collider
}

\author{
V. P. Gonçalves, ${ }^{1}$ M. V. T. Machado, ${ }^{2}$ F. S. Navarra, ${ }^{3}$ and D. Spiering ${ }^{3}$ \\ ${ }^{1}$ Instituto de Física e Matemática, Universidade Federal de Pelotas, C.P. 354, CEP 96010-900, Pelotas, Rio Grande do Sul, Brazil \\ ${ }^{2}$ High Energy Physics Phenomenology Group, GFPAE IF-UFRGS \\ Caixa Postal 15051, CEP 91501-970, Porto Alegre, Rio Grande do Sul, Brazil \\ ${ }^{3}$ Instituto de Física, Universidade de São Paulo, C.P. 66318, 05315-970, São Paulo, São Paulo, Brazil
}

(Received 30 August 2017; published 18 January 2018)

\begin{abstract}
The experimental results of the future Electron-Ion $(e A)$ Collider are expected to constrain the dynamics of the strong interactions at small values of the Bjorken $x$ variable and large nuclei. Recently it has been suggested that Coulomb corrections can be important in inclusive and diffractive $e A$ interactions. In this paper, we present a detailed investigation of the impact of the Coulomb corrections to some of the observables that will be measured in the future $e A$ collider. In particular, we estimate the magnitude of these corrections for the charm and longitudinal cross sections in inclusive and diffractive interactions. Our results demonstrate that the Coulomb corrections for these observables are negligible, which implies that they can be used to probe the QCD dynamics.
\end{abstract}

DOI: 10.1103/PhysRevC.97.015204

\section{INTRODUCTION}

The future Electron-Ion Collider (EIC) [1-4] will allow us to study the hadronic structure in the regime of large partonic densities and strong field strengths, which are expected to modify the linear evolution equations $[5,6]$ normally used in quantum chromodynamics (QCD) at high energies. We expect that the EIC experimental data will be able to establish the presence of gluon saturation effects and the magnitude of the associated nonlinear corrections and determine the correct theoretical framework for their description (for reviews see, e.g., Ref. [7]). In particular, the enhancement of nonlinear effects with the nuclear mass number has motivated the development of several studies on the implications of these effects in inclusive and exclusive observables which could be measured in electronion $(e A)$ colliders [8-25]. These studies have demonstrated that an $e A$ collider is the ideal environment to improve our undestanding of the strong interactions at high energies.

Deep inelastic $e A$ scattering is characterized by a large electron energy loss $v$ (in the target rest frame) and an invariant momentum transfer $q^{2} \equiv-Q^{2}$ between the incoming and outgoing electron such that $x=Q^{2} / 2 m_{N} v$ is fixed $\left(m_{N}\right.$ is the target mass). In terms of Fock states, we then view $e A$ scattering as follows: The electron emits a photon $(|e\rangle \rightarrow|e \gamma\rangle)$ with $E_{\gamma}=v$ and $p_{t \gamma}^{2} \approx Q^{2}$. Afterward, the photon splits into a $q \bar{q}$ pair $(|e \gamma\rangle \rightarrow|e q \bar{q}\rangle)$, which has electric and color charges, and typically travels a distance $l_{c} \approx 1 / m_{N} x$, referred to as the coherence length, before interacting in the target. For

Published by the American Physical Society under the terms of the Creative Commons Attribution 4.0 International license. Further distribution of this work must maintain attribution to the author $(s)$ and the published article's title, journal citation, and DOI. Funded by $S C O A P^{3}$. small $x$, the photon is converted into a $q \bar{q}$ pair at a large distance before the scattering. Consequently, the space-time picture of the Deep Inelastic Scattering (DIS) in the target rest frame can be viewed as the decay of the virtual photon at high energy into a quark-antiquark pair, which subsequently interacts with the target. If the target is a large- $A$ nucleus, it is characterized by a strong color field, generated by the fusion of the color fields of its nucleons. Consequently, in order to describe the dipole-nucleus scattering, one must take into account the multiple interactions of the dipole with the nuclear color field [7]. This aspect has been considered in the phenomenological works that have studied the impact of the nonlinear corrections to the QCD dynamics on the observables that will be measured in the future $e A$ collider [8-25]. However, these studies have not taken into account the contribution of the Coulomb corrections to the dipole-nucleus interaction. As the $q \bar{q}$ dipole has electric charge and a large nucleus generates a strong electromagnetic field, we might expect that the nuclear DIS receives a substantial contribution from electromagnetic interactions of the dipole with the nucleus, which is known as Coulomb correction. A first analysis of the impact of these corrections to the DIS off a heavy nucleus has been performed in Ref. [26] (see also Ref. [27]) and has indicated that the Coulomb corrections reach $20 \%$ of the inclusive cross section and about $40 \%$ of the diffractive cross section in the kinematical range of small values of the Bjorken $x$ variable and low photon virtualities $Q^{2}$. Considering the large magnitude predicted for these corrections, it is important to investigate in more detail how the behavior of different observables will be modified by the Coulomb corrections in the kinematical range that will be probed in the future EIC. Apart from the total inclusive and diffractive cross sections discussed in Ref. [26], the EIC is expected to measure the heavy quark and longitudinal contributions to these cross sections. In this paper, we extend the study performed in Ref. [26] and analyze the impact of the Coulomb corrections on these 
quantities. Our goal is to verify if it is possible to disentangle the Coulomb corrections from the QCD interactions in these different observables and, consequently, to probe the QCD dynamics at high energies.

This paper is organized as follows. In the next section, we briefly review the description of the photon-nucleus interaction in the dipole approach and establish the relation between the measured inclusive and diffractive cross sections and the colorelectric dipole target elastic scattering amplitude. Moreover, the treatment of the strong and electromagnetic interactions in the nuclear field is presented. In Sec. III, we present a detailed study of the Coulomb corrections to the charm and longitudinal contributions to the inclusive and diffractive cross sections. Our focus is on predictions for DIS off a heavy nucleus in the kinematical region of small values of $x$ and $Q^{2}$. Finally, in Sec. IV, we summarize our main results and conclusions.

\section{THEORETICAL FRAMEWORK}

The basic idea of the dipole approach is that the electronnucleus interaction at high energies can be factorized in terms of the fluctuation of the virtual photon into a $q \bar{q}$ dipole and the dipole-nucleus scattering. As a consequence, the longitudinal and transverse $\gamma^{*} A$ cross sections can be expressed as follows [28],

$$
\begin{aligned}
\sigma_{L, T}^{\mathrm{inc}}\left(x, Q^{2}\right)= & \sum_{f} \int d z d^{2} \boldsymbol{r}\left|\Psi_{L, T}^{(f)}\left(z, \boldsymbol{r}, Q^{2}\right)\right|^{2} \\
& \times 2 \int d^{2} \boldsymbol{b} \mathcal{N}(x, \boldsymbol{r}, \boldsymbol{b}),
\end{aligned}
$$

where the variable $\boldsymbol{r}$ defines the relative transverse separation of the pair (dipole) and $z(1-z)$ is the longitudinal momentum fraction of the quark (antiquark) of flavor $f$. In the case of diffractive $e A$ collisions, the dipole approach predicts that the cross sections can be expressed as follows:

$$
\begin{aligned}
\sigma_{L, T}^{\mathrm{diff}}\left(x, Q^{2}\right)= & \sum_{f} \int d z d^{2} \boldsymbol{r}\left|\Psi_{L, T}^{(f)}\left(z, \boldsymbol{r}, Q^{2}\right)\right|^{2} \\
& \times \int d^{2} \boldsymbol{b}|\mathcal{N}(x, \boldsymbol{r}, \boldsymbol{b})|^{2} .
\end{aligned}
$$

The photon wave functions $\Psi_{L, T}$ are determined from light cone perturbation theory and are given by

$$
\begin{aligned}
\left|\Psi_{T}\left(z, \boldsymbol{r}, Q^{2}\right)\right|^{2}= & \frac{6 \alpha_{\mathrm{em}}}{4 \pi^{2}} \sum_{f} e_{f}^{2}\left[z^{2}+(1-z)^{2}\right] \varepsilon^{2} K_{1}^{2}(\varepsilon r) \\
& +m_{f}^{2} K_{0}^{2}(\varepsilon r)
\end{aligned}
$$

and

$$
\left|\Psi_{L}\left(z, \boldsymbol{r}, Q^{2}\right)\right|^{2}=\frac{6 \alpha_{\mathrm{em}}}{\pi^{2}} \sum_{f} e_{f}^{2}\left\{Q^{2} z^{2}(1-z)^{2} K_{0}^{2}(\varepsilon r)\right\} .
$$

The auxiliary variable $\varepsilon^{2}=z(1-z) Q^{2}+m_{f}^{2}$ depends on the quark mass, $m_{f}$. The $K_{0,1}$ are the McDonald functions and the summation is performed over the quark flavors. Here, we consider the light quark masses to be $m_{f}=0.14 \mathrm{GeV}$ (for $f=u, d, s)$ and for the heavy ones we consider $m_{c}=1.4 \mathrm{GeV}$ and $m_{b}=4.5 \mathrm{GeV}$.
The function $\mathcal{N}(x, \boldsymbol{r}, \boldsymbol{b})$ is the quark dipole-nucleus forward scattering amplitude for a given impact parameter $\boldsymbol{b}$ which encodes all the information about the scattering due to strong and electromagnetic interactions. In order to estimate this quantity, we will consider the Glauber multiple scattering approach $[29,30]$, which is justified at high energies when the incident particle passes through the nucleus in a very short time, so that the change of the positions of the nucleons can be neglected. In this model, the scattering amplitude obtained by calculating the scattering from instantaneously fixed nucleons is then averaged over the initial and final nucleus wave functions. The main assumption is that the total eikonal phase acquired by the particle when passing through the nucleus at a fixed impact parameter is equal to the sum of the phases from the individual nucleons. During recent decades, the Glauber model has been successfully applied to describe proton-nucleus collisions (for a review, see, e.g., Ref. [31]). In particular, the impact of the Coulomb corrections has been studied by several authors (see, e.g., Refs. [30,32-34]), who have demonstrated that it plays an important role in heavy nuclei, influencing the differential cross sections around the minima of the squared momentum transfer $(t)$ distributions. The basic assumption in these studies is that the proton-nucleon phase shift can be described by the sum of purely strong and Coulomb phase shifts [30,32,33]. In Refs. [26,35,36], the Glauber model has been applied to describe the dipole-nucleus interaction and the additivity of the strong and Coulomb phase shifts to estimate the dipole-nucleon was assumed. As a consequence, the dipole-nucleus forwardscattering amplitude can be expressed as follows [26,36]:

$$
\mathcal{N}(x, \boldsymbol{r}, \boldsymbol{b})=1-\cos \left[Z\left\langle\operatorname{Re} i \Gamma_{\mathrm{em}}\right\rangle\right] \exp \left[-A\left\langle\operatorname{Im} i \Gamma_{s}\right\rangle\right],
$$

where $i \Gamma_{\mathrm{em}}$ and $i \Gamma_{s}$ are the electromagnetic and strong contributions to the dipole-nucleon elastic scattering amplitude, respectively. At large impact parameters $\left(b>R_{A}\right)$, the strong interaction contribution is expected to be small, i.e., $A\left[i \Gamma_{s}\right] \rightarrow 0$, and hence

$$
\mathcal{N}(x, \boldsymbol{r}, \boldsymbol{b}) \approx \mathcal{N}_{\mathrm{em}}(x, \boldsymbol{r}, \boldsymbol{b})=1-\cos \left[Z\left\langle i \Gamma_{\mathrm{em}}\right\rangle\right] .
$$

On the other hand, at small impact parameters $\left(b<R_{A}\right)$, the strong interaction becomes dominant and the dipole-nucleus scattering amplitude can be approximated by

$$
\mathcal{N}(x, \boldsymbol{r}, \boldsymbol{b}) \approx \mathcal{N}_{s}(x, \boldsymbol{r}, \boldsymbol{b})=1-\exp \left[-A\left\langle i \Gamma_{s}\right\rangle\right] .
$$

In what follows, we will assume that these asymptotic solutions can be used to estimate the dipole-nucleus scattering amplitude for $b \sim R_{A}$, which is the region of interest for the deep inelastic scattering. In order to calculate the inclusive and diffractive cross sections, we must specify the models used for the electromagnetic and strong dipole-nucleus scattering amplitude. Following Ref. [26], we will assume that

$$
\mathcal{N}_{s}(x, \boldsymbol{r}, \boldsymbol{b})=\left[1-\exp \left(-\frac{Q_{\mathrm{s}, A}^{2}(x) \boldsymbol{r}^{2}}{4}\right)\right] \Theta\left(R_{A}-b\right),
$$

where $Q_{\mathrm{s}, A}$ is the nuclear saturation scale, which we will assume to be given by $Q_{\mathrm{s}, A}^{2}(x)=A^{1 / 3} Q_{\mathrm{s}}^{2}$, where $Q_{\mathrm{s}}^{2}(x)=$ $Q_{0}^{2} e^{\lambda \ln \left(x_{0} / x\right)}$ is the saturation scale for a proton. This model is a naive generalization for the nucleus of the saturation model proposed in Refs. [37], which captures the main properties 

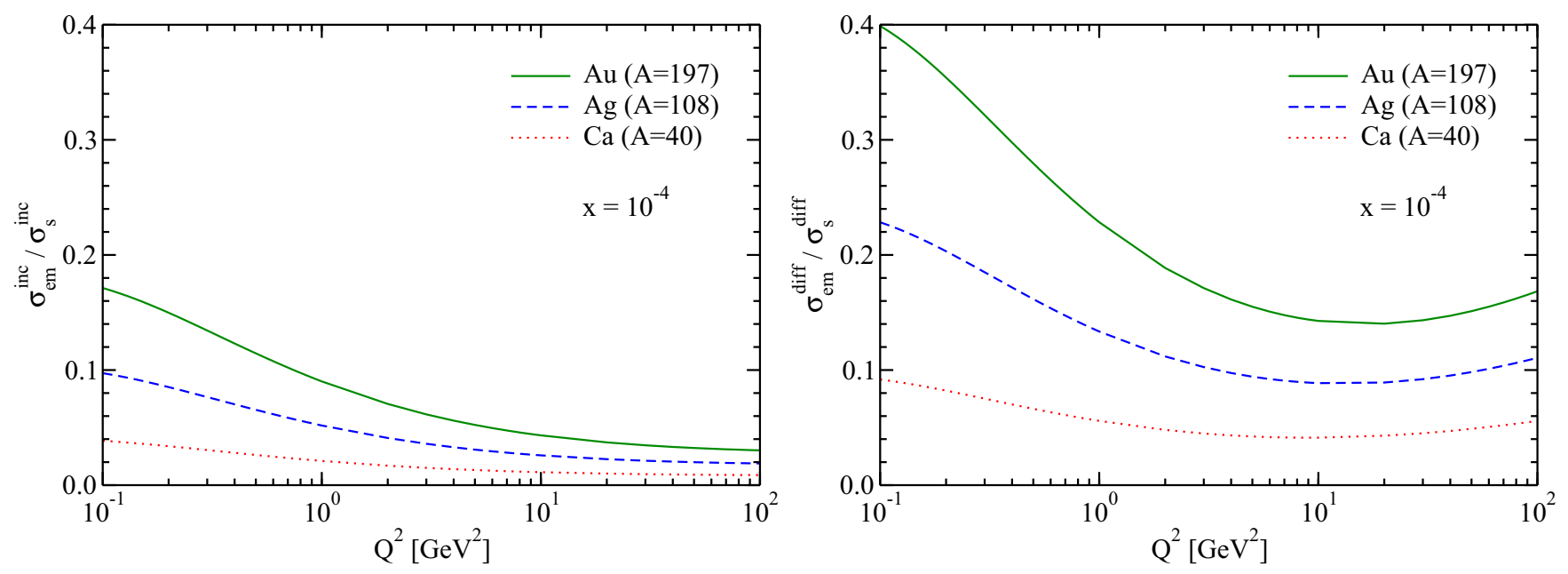

FIG. 1. Dependence on the photon virtuality $Q^{2}$ for different atomic numbers and $x=10^{-4}$ of the ratio between the electromagnetic and strong contributions for the inclusive (left panel) and diffractive (right panel) cross sections.

of the high-energy evolution equations and it is suitable for describing the nonlinear physics in the small- $x$ region. In particular, this model implies that $\mathcal{N}_{s} \propto r^{2}$ (color transparency) at small pair separations and that the multiple scatterings are resumed in a Glauber-inspired way. Such a model was used in Refs. $[9,10]$ to estimate the impact of the saturation physics in the observables that will be measured in the future $e A$ collider. In our calculations we will consider the parameters obtained from a fit to the HERA data in Ref. [37]: $Q_{0}^{2}=1 \mathrm{GeV}^{2}, \lambda=$ 0.277 , and $x_{0}=3.41 \times 10^{-4}$. Using Eq. (8), we obtain that

$$
\begin{aligned}
2 \int_{b<R_{A}} d^{2} \boldsymbol{b} \mathcal{N}(x, \boldsymbol{r}, \boldsymbol{b}) & \approx 2 \int_{b<R_{A}} d^{2} \boldsymbol{b} \mathcal{N}_{s}(x, \boldsymbol{r}, \boldsymbol{b}) \\
& =2 \pi R_{A}^{2}\left(1-e^{-Q_{\mathrm{s}, A}^{2} r^{2} / 4}\right)
\end{aligned}
$$

and

$$
\begin{aligned}
\int_{b<R_{A}} d^{2} \boldsymbol{b}|\mathcal{N}(x, \boldsymbol{r}, \boldsymbol{b})|^{2} & \approx \int_{b<R_{A}} d^{2} \boldsymbol{b}\left|\mathcal{N}_{s}(x, \boldsymbol{r}, \boldsymbol{b})\right|^{2} \\
& =\pi R_{A}^{2}\left(1-e^{-Q_{s}^{2} r^{2} / 4}\right)^{2}
\end{aligned}
$$

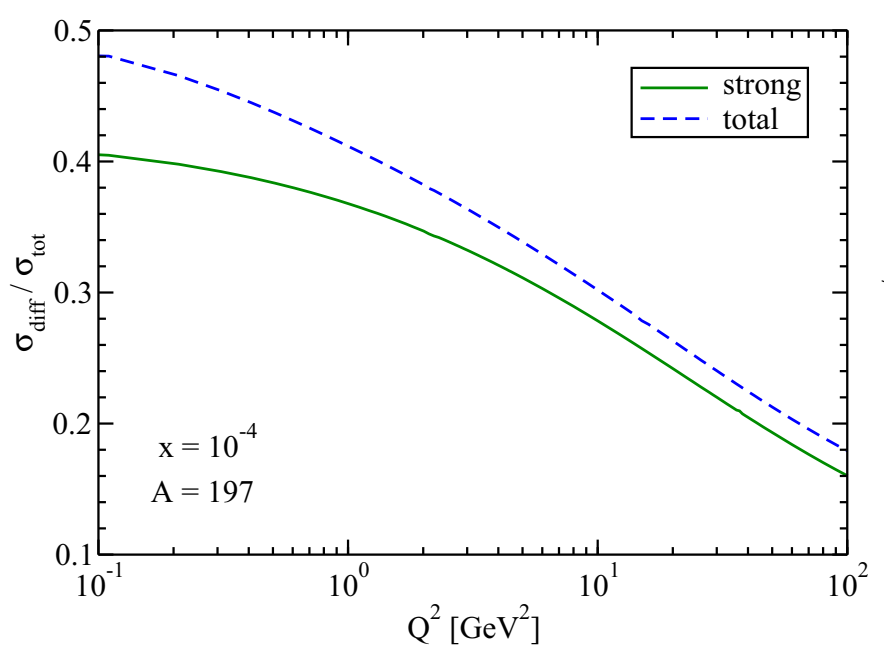

For the electromagnetic case, we will follow the approach proposed in Refs. [26,36]. In particular, we will assume that the leading term in $\alpha$ for the dipole-nucleon scattering arises from a single-photon exchange, with the elastic dipole-nucleon amplitude being approximately real. As demonstrated in Ref. [35], at the Born approximation, the contribution of the imaginary part is proportional to $\alpha^{2} Z \sim \alpha$ in the $Z \alpha \sim 1$, while the contribution of the real part is of the order of $\alpha^{2} Z^{2} \sim$ 1. Considering the Weizscker-Williams approximation, where the $t$-channel photons at high energies are assumed to be almost real, the electromagnetic dipole-nucleon elastic scattering amplitude was derived in Ref. [35], given by

$$
\left\langle i \Gamma_{\mathrm{em}}\right\rangle \approx 2 \alpha \ln \left(\frac{|\boldsymbol{b}-\boldsymbol{r} / 2|}{|\boldsymbol{b}+\boldsymbol{r} / 2|}\right)=\alpha \ln \left(\frac{b^{2}+r^{2}-b r \cos \phi}{b^{2}+r^{2}+b r \cos \phi}\right) .
$$

Moreover, we will take into account that for the deep inelastic scattering the main contributions for the DIS cross sections comes from values of pair separations of the order $r \sim 1 / m_{f}[9,10]$. Consequently, we will have that in general

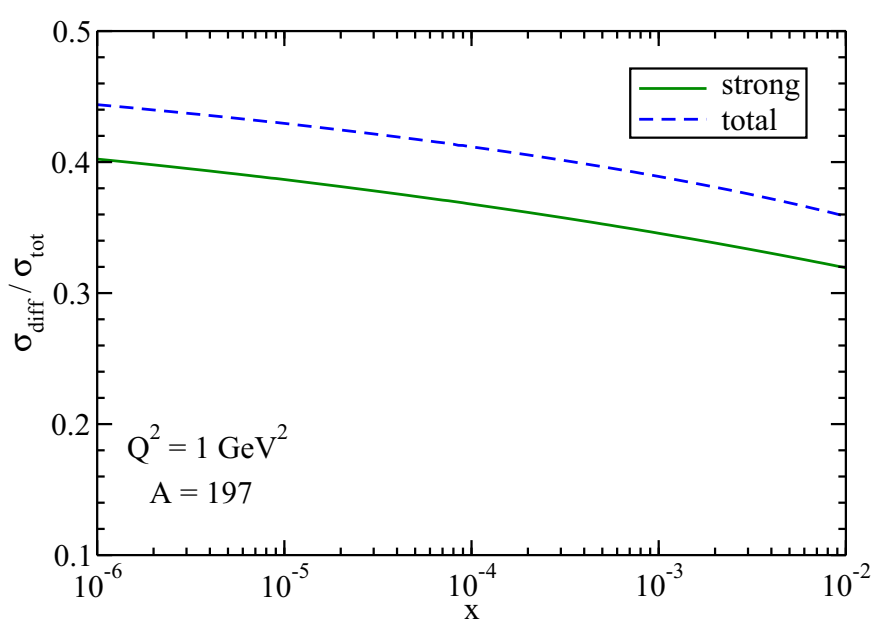

FIG. 2. Ratio $\sigma_{\text {diff }} / \sigma_{\text {tot }}$ as a function of $Q^{2}$ for fixed $x$ (left panel) and as a function of $x$ for fixed $Q^{2}$ (right panel). 

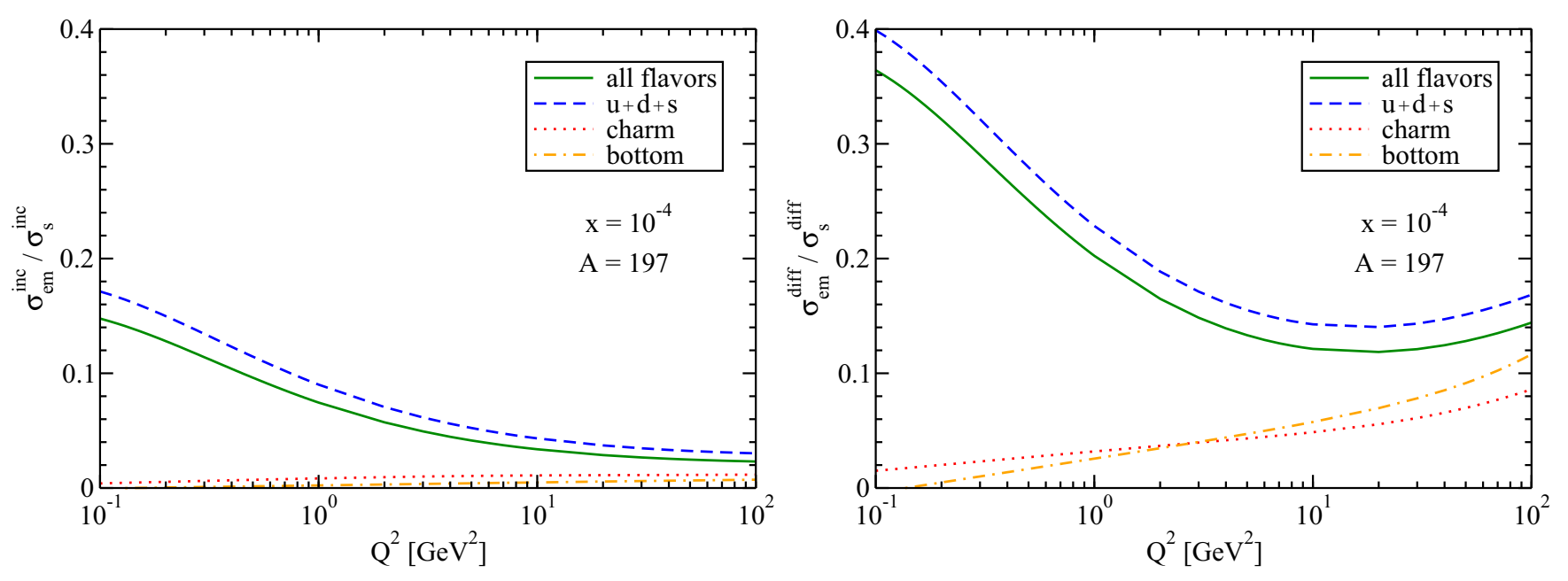

FIG. 3. Flavor decomposition for the ratio between the electromagnetic and strong contributions for the inclusive (left panel) and diffractive (right panel) processes as a function of photon virtuality $Q^{2}$ and fixed $x=10^{-4}$ for gold nucleus.

$r \ll b$, which allows us to simplify the expression for the dipole-nucleon scattering amplitude. Such inequality becomes stronger in the case of the heavy quarks. In particular, for $b \gg r$, Eq. (11) can be simplified and becomes

$$
\left\langle i \Gamma_{\mathrm{em}}\right\rangle=2 \alpha \frac{\boldsymbol{b} \cdot \boldsymbol{r}}{b^{2}}+\mathcal{O}\left(r^{3} / b^{3}\right) \approx \frac{2 \alpha r \cos \phi}{b},
$$

which implies that

$$
\begin{aligned}
\cos \left[Z\left\langle i \Gamma_{\mathrm{em}}\right\rangle\right] & =\cos \left(\frac{2 \alpha Z r \cos \phi}{b}\right) \\
& =1-\frac{1}{2}\left(\frac{2 \alpha Z r \cos \phi}{b}\right)^{2}+\mathcal{O}\left(r^{4} / b^{4}\right) \\
& \approx 1-\frac{2 \alpha^{2} Z^{2} r^{2} \cos ^{2} \phi}{b^{2}}
\end{aligned}
$$

and

$$
\mathcal{N}_{\mathrm{em}}(x, \boldsymbol{r}, \boldsymbol{b}) \approx \frac{2 \alpha^{2} Z^{2} r^{2} \cos ^{2} \phi}{b^{2}}
$$

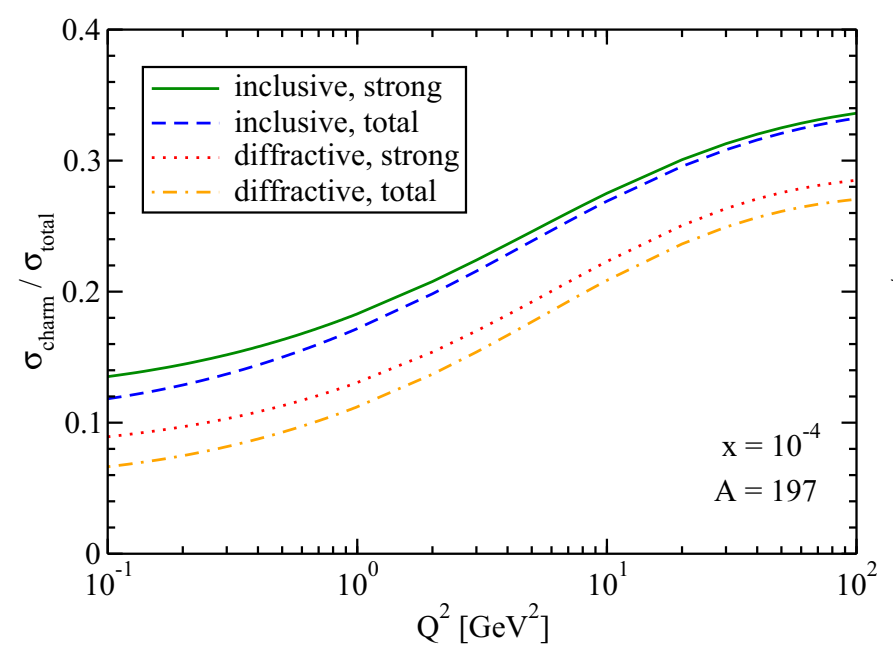

Consequently, we have

$$
\begin{aligned}
2 \int_{b>R_{A}} d^{2} \boldsymbol{b} \mathcal{N}(x, \boldsymbol{r}, \boldsymbol{b}) & \approx 2 \int_{b>R_{A}} d^{2} \boldsymbol{b} \mathcal{N}_{\mathrm{em}}(x, \boldsymbol{r}, \boldsymbol{b}) \\
& =4 \alpha^{2} Z^{2} r^{2} \int_{R_{A}}^{b_{\max }} \frac{d b}{b} \int_{0}^{2 \pi} d \phi \cos ^{2} \phi \\
& =4 \pi \alpha^{2} Z^{2} r^{2} \ln \left(\frac{b_{\max }}{R_{A}}\right) \\
& =4 \pi \alpha^{2} Z^{2} r^{2} \ln \left(\frac{W^{2}}{4 m_{f}^{2} m_{N} R_{A}}\right)
\end{aligned}
$$

where $m_{N}$ is the nucleon mass and $W$ is the photon-nucleus center-of-mass energy. Moreover, $b_{\max }=s /\left(4 m_{N} m_{f}^{2}\right)$ is a long-distance cutoff of the $b$ integral, which is directly related to the minimum transverse momentum at which the Weizsacker-Williams approximation still is valid [35].

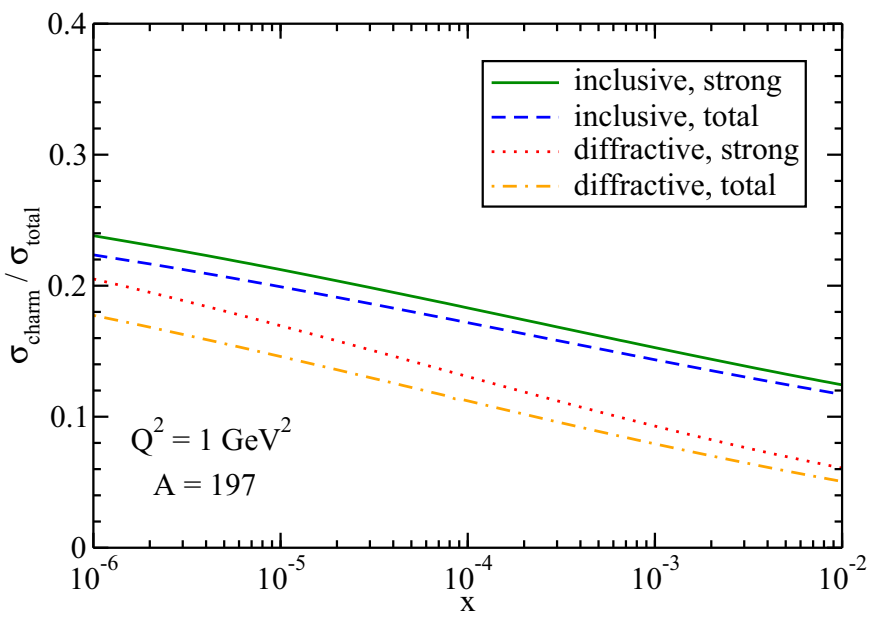

FIG. 4. Dependence on $Q^{2}$ (left panel) and $x$ (right panel) of the ratios between the charm and total cross sections for inclusive and diffractive interactions. 

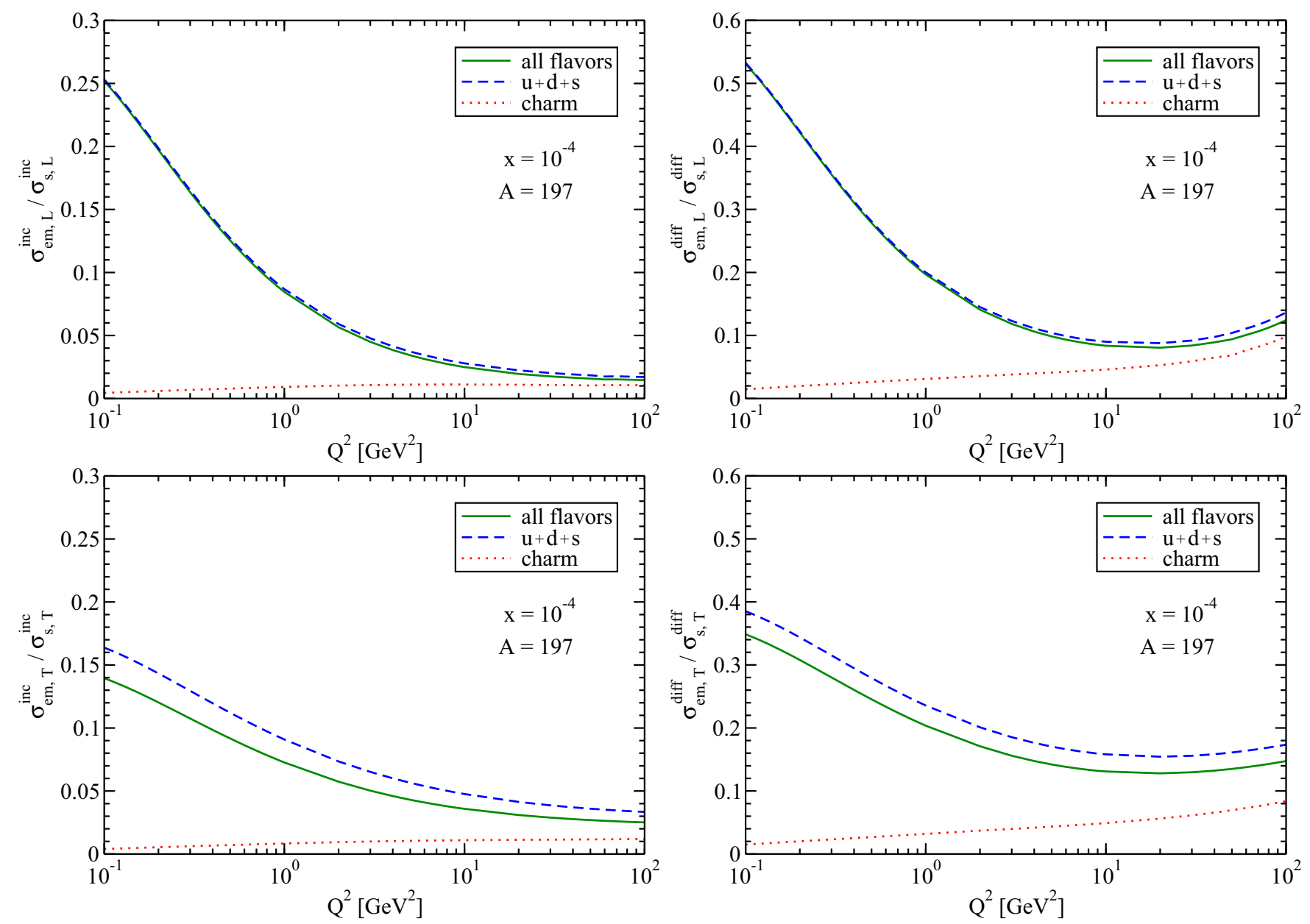

FIG. 5. Upper panels: Dependence on $Q^{2}$ of the ratio between the electromagnetic and strong longitudinal cross sections for the inclusive (left) and diffractive (right) interactions. Lower panels: Dependence on $Q^{2}$ of the ratio between the electromagnetic and strong transverse cross sections for inclusive (left) and diffractive (right) interactions.

As in demonstrated in Ref. [35], one has that $b_{\max } \gg R_{A}$, which justifies the approximations used in the derivation of Eqs. (11)-(16). Considering the same approximations, for $b>R_{A}$ we find

$$
\begin{aligned}
|\mathcal{N}(x, \boldsymbol{r}, \boldsymbol{b})|^{2} & \approx\left|\mathcal{N}_{\mathrm{em}}(x, \boldsymbol{r}, \boldsymbol{b})\right|^{2}=\left|1-\cos \left[Z\left\langle\operatorname{Re} i \Gamma_{\mathrm{em}}\right\rangle\right]\right|^{2} \\
& =1-2 \cos \left[Z\left\langle\operatorname{Re} i \Gamma_{\mathrm{em}}\right\rangle\right]+\cos ^{2}\left[Z\left\langle\operatorname{Re} i \Gamma_{\mathrm{em}}\right\rangle\right] .
\end{aligned}
$$

Using $\cos ^{2}\left[Z\left\langle i \Gamma_{\mathrm{em}}\right\rangle\right]=1+\mathcal{O}\left(\alpha^{4} r^{4} / b^{4}\right)$ yields

$$
\left|\mathcal{N}_{\mathrm{em}}(x, \boldsymbol{r}, \boldsymbol{b})\right|^{2} \approx \frac{4 \alpha^{2} Z^{2} r^{2} \cos ^{2} \phi}{b^{2}},
$$

which implies that

$$
\begin{aligned}
\int_{b>R_{A}} d^{2} \boldsymbol{b}|\mathcal{N}(x, \boldsymbol{r}, \boldsymbol{b})|^{2} & \approx \int_{b>R_{A}} d^{2} \boldsymbol{b}\left|\mathcal{N}_{\mathrm{em}}(x, \boldsymbol{r}, \boldsymbol{b})\right|^{2} \\
& =4 \pi \alpha^{2} Z^{2} r^{2} \ln \left(\frac{W^{2}}{4 m_{f}^{2} m_{N} R_{A}}\right) .
\end{aligned}
$$

As obtained in Ref. [26], the electromagnetic contribution for the inclusive and diffractive cross sections are equal, differently from the strong one. As a consequence, we expect that its impact on inclusive and diffractive observables will be different. In the next section, we will estimate how the electromagnetic contribution modifies these observables in the kinematical range that will be probed by the future Electron-Ion Collider.

\section{RESULTS AND DISCUSSION}

In this section, we present a detailed study of the flavor and polarization decomposition of inclusive and diffractive cross sections including the Coulomb correction. Improving on the work done in Ref. [26], we will include in our analysis the contribution of the heavy quarks (charm and bottom). As discussed, e.g., in Refs. $[9,15]$, and shown in what follows, the charm contribution is expected to be $\approx 20 \%$ of the total cross section at small values of $x$ and low virtualities. Moreover, it is considered an important probe of the nuclear gluon distribution. Similar expectation also motivates the analysis of the longitudinal cross section (see, e.g., Refs. [14,38]). Our goal is to verify the impact of the Coulomb corrections in these observables in inclusive and diffractive interactions.

We start our analysis by presenting in Fig. 1 the ratio between the Coulomb and strong (QCD) contributions to 
the inclusive (left panel) and diffractive (right panel) cross sections. In Fig. 1, the ratios are shown as a function of photon virtuality $Q^{2}$ for a fixed value of Bjorken variable, $x=10^{-4}$, and three distinct nuclei: gold (solid line), silver (dashed line), and calcium (dotted line). The results are consistent with those obtained in Ref. [26], even including the heavy quark contribution, showing that the Coulomb correction is quite important in the kinematical region of low $Q^{2}$ and small $x$ and it is enhanced for the heavier nuclei. In the perturbative range that will be probed in the future $\operatorname{EIC}\left(Q^{2} \gtrsim 1 \mathrm{GeV}^{2}\right)$ and $A=$ 197 , the Coulomb contribution is $\approx 10(21) \%$ for the inclusive (diffractive) cross section. The different impact of the Coulomb interactions on the inclusive and diffractive cross sections can be understood as follows. In contrast to the inclusive cross section, the main contribution to the diffractive cross section comes from larger dipoles (see, e.g., Ref. [10]) and the electromagnetic terms are proportional to $r^{2}$ [see Eqs. (16) and (19)]. As a consequence, the presence of Coulomb corrections modifies the ratio between the diffractive and the total cross section, which is expected to be measured in the future Electron-Ion Collider. Our predictions for the $Q^{2}$ and $x$ dependence of this ratio are presented in Fig. 2. We compare the predictions obtained with the sum of the electromagnetic and strong contributions, denoted total in the figure, with those derived disregarding the Coulomb corrections. We observe that the ratio is enhanced by the electromagnetic contribution, in particular at low $Q^{2}$ and small $x$. At $Q^{2}=1 \mathrm{GeV}^{2}$, this enhancement is $\approx 10 \%$.

The $r^{2}$ dependence of the electromagnetic contribution has a direct impact on the Coulomb corrections to the heavy quark cross sections. As these cross sections are dominated by small dipoles, we expect that the effect of the Coulomb corrections will be smaller here than in the case of light quark production. This expectation is confirmed by the results shown in Fig. 3, where we present the flavor decomposition of the ratio between the electromagnetic and strong contributions. We observe that for $Q^{2} \approx 1 \mathrm{GeV}^{2}$ the Coulomb corrections can be disregarded in the charm and bottom production in the inclusive case and are of order of $2 \%$ in diffractive interactions. At larger values of $Q^{2}$, the heavy quark contribution to the total cross sections increases as well as the Coulomb corrections. We have verified that the impact of the Coulomb corrections to the charm and bottom production is almost $x$ independent in the range $1 \leqslant Q^{2} \leqslant 10 \mathrm{GeV}^{2}$. Another interesting aspect is that the inclusion of the heavy quarks decreases the magnitude of the Coulomb corrections for the total cross sections in comparison to those obtained considering only light quarks, denoted by $u+d+s$ in the figure.

Let us now analyze more carefully the impact of the Coulomb corrections on the charm cross section, which is expected to be measured in the future Electron-Ion Collider. In Fig. 4, we present our predictions for the $Q^{2}$ (left panel) and $x$ (right panel) dependence of the ratio between the charm and the total cross sections for inclusive and diffractive interactions. The predictions obtained disregarding the Coulomb corrections are denoted as strong in the figure. The charm contribution increases with $Q^{2}$ and at smaller values of $x$. Moreover, it is larger for inclusive processes. Our results indicate that the inclusion of the Coulomb corrections implies a mild decreasing of the ratios. In particular for inclusive processes, the $Q^{2}$ and $x$ dependence of the ratios are only slightly modified by Coulomb corrections in the perturbative $Q^{2}$ range that will probed in the future Electron-Ion Collider. This result implies that the study of the charm production is a good probe of the high-energy regime of the QCD dynamics.

Let us now consider the impact of the Coulomb corrections on the longitudinal and transverse cross sections in inclusive and diffractive interactions. In Fig. 5 (upper panels), we present our results for the ratio between the electromagnetic and strong longitudinal cross sections. In the left (right) panel, we show the inclusive (diffractive) cross sections. In the inclusive case, the ratio rapidly decreases with $Q^{2}$ and becomes smaller than $5 \%$ for $Q^{2} \gtrsim 1.5 \mathrm{GeV}^{2}$. In the diffractive case, the Coulomb correction is larger by a factor of 2 in the same kinematical range. As expected from our previous analysis, the charm contribution is small in the $1 \leqslant Q^{2} \leqslant 10 \mathrm{GeV}^{2}$ range. The results presented in the lower panels of Fig. 5 are analogous to those of the upper panels but refer to the transverse cross sections. They indicate that the Coulomb corrections to both longitudinal and
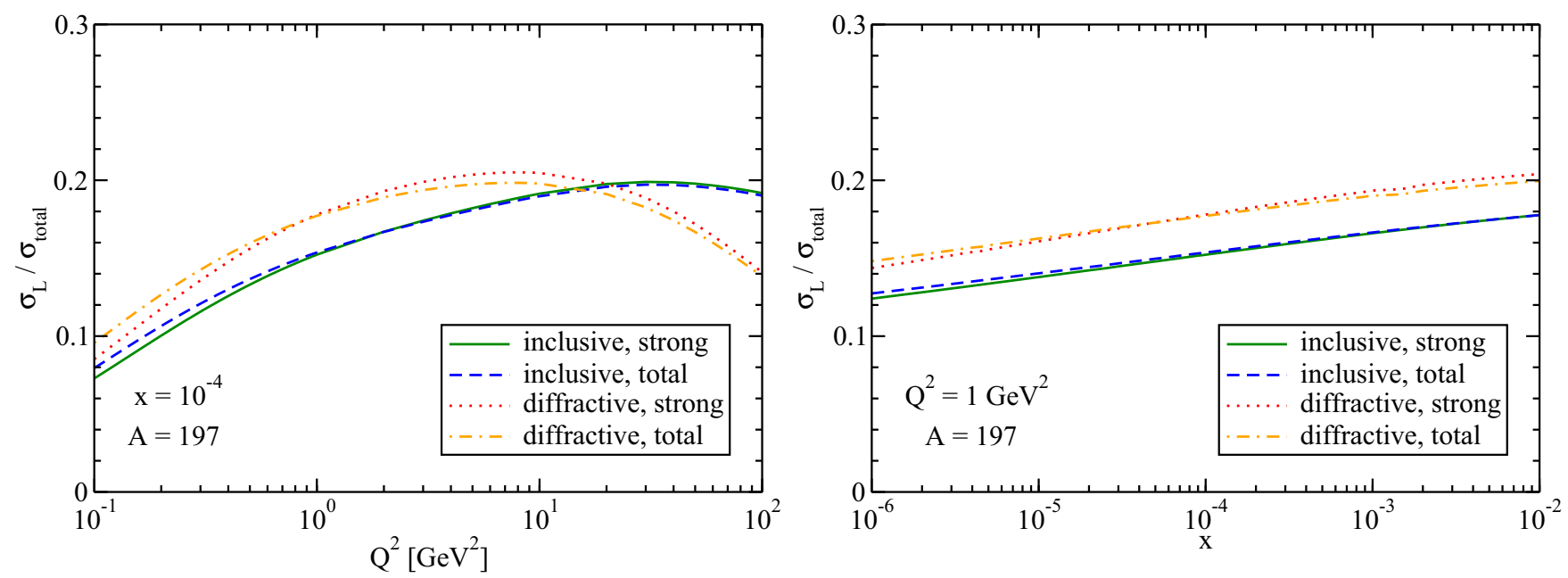

FIG. 6. Dependence on $Q^{2}$ (left panel) and $x$ (right panel) for the ratio between the longitudinal and total cross sections for inclusive and diffractive interactions. 
transverse cross sections are comparable. Moreover, compared to the strong cross sections, they are small everywhere, except in the very low $Q^{2}\left(Q^{2} \simeq 0.1 \mathrm{GeV}^{2}\right)$ region, where dipoles of larger size dominate the cross sections. In the figures, we also see that for the charm cross sections, the Coulomb corrections are very small. The fact that the longitudinal cross section is not sensitive to the Coulomb corrections is illustrated in a different way in Fig. 6, where we present the $Q^{2}$ and $x$ dependence of the ratio $\sigma_{L} / \sigma_{\text {tot }}$ in inclusive and diffractive interactions. These results demonstrate that the inclusion of the Coulomb corrections has no impact on the ratio. The same is true for the transverse cross sections. Therefore, the study of the longitudinal and transverse cross sections can also be useful to understand the QCD dynamics at small $x$.

A final comment is in order. In the analysis presented above, we have assumed, following Refs. [26,36], that the modeling of the strong dipole-nucleus scattering amplitude is given by Eq. (8). However, as discussed in Refs. [15,19], a more realistic model for $\mathcal{N}_{s}(x, \boldsymbol{r}, \boldsymbol{b})$ can be derived using the GlauberMueller (GM) approach [39]. In particular, in Ref. [15], two of the authors have demonstrated that the GM approach is able to describe the current data for the nuclear structure function. In order to verify the dependence of our predictions on the model used to describe $\mathcal{N}_{s}$ we also have estimated the different observables discussed in this section using the GM approach and have obtained that the contribution of the Coulomb corrections still are smaller than those presented here.

\section{SUMMARY}

In this paper, we have studied in detail the Coulomb contribution to some of the observables that will be measured in the future $e A$ collider. In particular, we study the impact of these corrections on the total, charm, and longitudinal cross sections of inclusive and diffractive interactions. Our analysis is motivated by the fact that these observables are expected to probe the QCD dynamics and constrain the description of the nonlinear effects. Our results indicate that the Coulomb corrections to the total cross sections are important at low $Q^{2}$ and small values of $x$ and are larger for diffractive interactions. In particular, the ratio between the diffractive and total cross sections are enhanced by $\approx 10 \%$. In contrast, our results indicate the impact of the Coulomb corrections on the transverse and longitudinal cross sections is small and on the charm cross sections is negligible. Therefore, these observables (especially the latter) can be considered clean probes of the QCD dynamics.

\section{ACKNOWLEDGMENTS}

The authors thank Kirill Tuchin for helpful comments. This work was partially financed by the Brazilian funding agencies CNPq, CAPES, FAPERGS, FAPESP (Contract No. 12/509844), and INCT-FNA (Process No. 464898/2014-5).
[1] A. Deshpande, R. Milner, R. Venugopalan, and W. Vogelsang, Ann. Rev. Nucl. Part. Sci. 55, 165 (2005).

[2] D. Boer, M. Diehl, R. Milner, R. Venugopalan, W. Vogelsang, D. Kaplan, H. Montgomery, S. Vigdor et al., arXiv:1108.1713 [nucl-th].

[3] A. Accardi, J. L. Albacete, M. Anselmino, N. Armesto, E. C. Aschenauer, A. Bacchetta, D. Boer, W. Brooks et al., Eur. Phys. J. A 52, 268 (2016).

[4] E. C. Aschenauer et al., arXiv:1708.01527 [nucl-ex].

[5] V. N. Gribov and L. N. Lipatov, Sov. J. Nucl. Phys. 15, 438 (1972); G. Altarelli and G. Parisi, Nucl. Phys. B 126, 298 (1977); Yu. L. Dokshitzer, Sov. Phys. JETP 46, 641 (1977).

[6] L. N. Lipatov, Sov. J. Nucl. Phys. 23, 338 (1976); E. A. Kuraev, L. N. Lipatov, and V. S. Fadin, Sov. Phys. JETP 44, 443 (1976); 45, 199 (1977); I. I. Balitsky and L. N. Lipatov, Sov. J. Nucl. Phys. 28, 822 (1978).

[7] F. Gelis, E. Iancu, J. Jalilian-Marian, and R. Venugopalan, Ann. Rev. Nucl. Part. Sci. 60, 463 (2010); E. Iancu and R. Venugopalan, in Quark Gluon Plasma 3, edited by R. C. Hwa and X.-N. Wang (World Scientific, Singapore, 2004); H. Weigert, Prog. Part. Nucl. Phys. 55, 461 (2005); J. Jalilian-Marian and Y. V. Kovchegov, ibid. 56, 104 (2006); J. L. Albacete and C. Marquet, ibid. 76, 1 (2014).

[8] V. P. Goncalves, Phys. Lett. B 495, 303 (2000).

[9] M. S. Kugeratski, V. P. Goncalves, and F. S. Navarra, Eur. Phys. J. C 46, 465 (2006).

[10] M. S. Kugeratski, V. P. Goncalves, and F. S. Navarra, Eur. Phys. J. C 46, 413 (2006)

[11] N. N. Nikolaev, W. Schafer, B. G. Zakharov, and V. R. Zoller, JETP Lett. 84, 537 (2007).
[12] H. Kowalski, T. Lappi, and R. Venugopalan, Phys. Rev. Lett. 100, 022303 (2008)

[13] H. Kowalski, T. Lappi, C. Marquet, and R. Venugopalan, Phys. Rev. C 78, 045201 (2008).

[14] E. R. Cazaroto, F. Carvalho, V. P. Goncalves, and F. S. Navarra, Phys. Lett. B 669, 331 (2008).

[15] E. R. Cazaroto, F. Carvalho, V. P. Goncalves, and F. S. Navarra, Phys. Lett. B 671, 233 (2009).

[16] V. P. Goncalves, M. S. Kugeratski, M. V. T. Machado, and F. S. Navarra, Phys. Rev. C 80, 025202 (2009).

[17] V. P. Goncalves, M. S. Kugeratski, and F. S. Navarra, Phys. Rev. C 81, 065209 (2010).

[18] A. Caldwell and H. Kowalski, Phys. Rev. C 81, 025203 (2010).

[19] E. R. Cazaroto, F. Carvalho, V. P. Goncalves, M. S. Kugeratski, and F. S. Navarra, Phys. Lett. B 696, 473 (2011).

[20] T. Lappi and H. Mantysaari, Phys. Rev. C 83, 065202 (2011).

[21] T. Toll and T. Ullrich, Phys. Rev. C 87, 024913 (2013).

[22] J. T. Amaral, V. P. Goncalves, and M. S. Kugeratski, Nucl. Phys. A 930, 104 (2014).

[23] T. Lappi, H. Mantysaari, and R. Venugopalan, Phys. Rev. Lett. 114, 082301 (2015).

[24] V. P. Gonçalves and D. S. Pires, Phys. Rev. C 91, 055207 (2015).

[25] V. P. Goncalves, F. S. Navarra, and D. Spiering, J. Phys. G 43, 095002 (2016).

[26] K. Tuchin, Phys. Rev. Lett. 112, 072001 (2014).

[27] B. Z. Kopeliovich, A. V. Tarasov, and O. O. Voskresenskaya, Eur. Phys. J. A 11, 345 (2001).

[28] N. N. Nikolaev and B. G. Zakharov, Z. Phys. C 49, 607 (1991); 53, 331 (1992); A. H. Mueller, Nucl. Phys. B 415, 373 (1994); 425, 471 (1994). 
[29] R. J. Glauber, Lectures in Theoretical Physics, edited by W. E. Brittin and L. G. Duham (Interscience, New York, 1959), Vol. 1.

[30] R. J. Glauber and G. Matthiae, Nucl. Phys. B 21, 135 (1970).

[31] G. D. Alkhazov, S. L. Belostotsky, and A. A. Vorobev, Phys. Rep. 42, 89 (1978).

[32] W. Czyz, L. Lesniak, and H. Wolek, Nucl. Phys. B 19, 125 (1970).

[33] H. Lesniak and L. Lesniak, Nucl. Phys. B 38, 221 (1972).
[34] M. Bleszynski and T. Jaroszewicz, Nucl. Phys. A 256, 429 (1976).

[35] K. Tuchin, Phys. Rev. D 80, 093006 (2009).

[36] K. Tuchin, Phys. Rev. C 89, 024904 (2014).

[37] K. Golec-Biernat and M. Wüsthoff, Phys. Rev. D 59, 014017 (1998); 60, 114023 (1999).

[38] E. C. Aschenauer, S. Fazio, M. A. C. Lamont, H. Paukkunen, and P. Zurita, Phys. Rev. D 96, 114005 (2017).

[39] A. H. Mueller, Nucl. Phys. B 335, 115 (1990). 